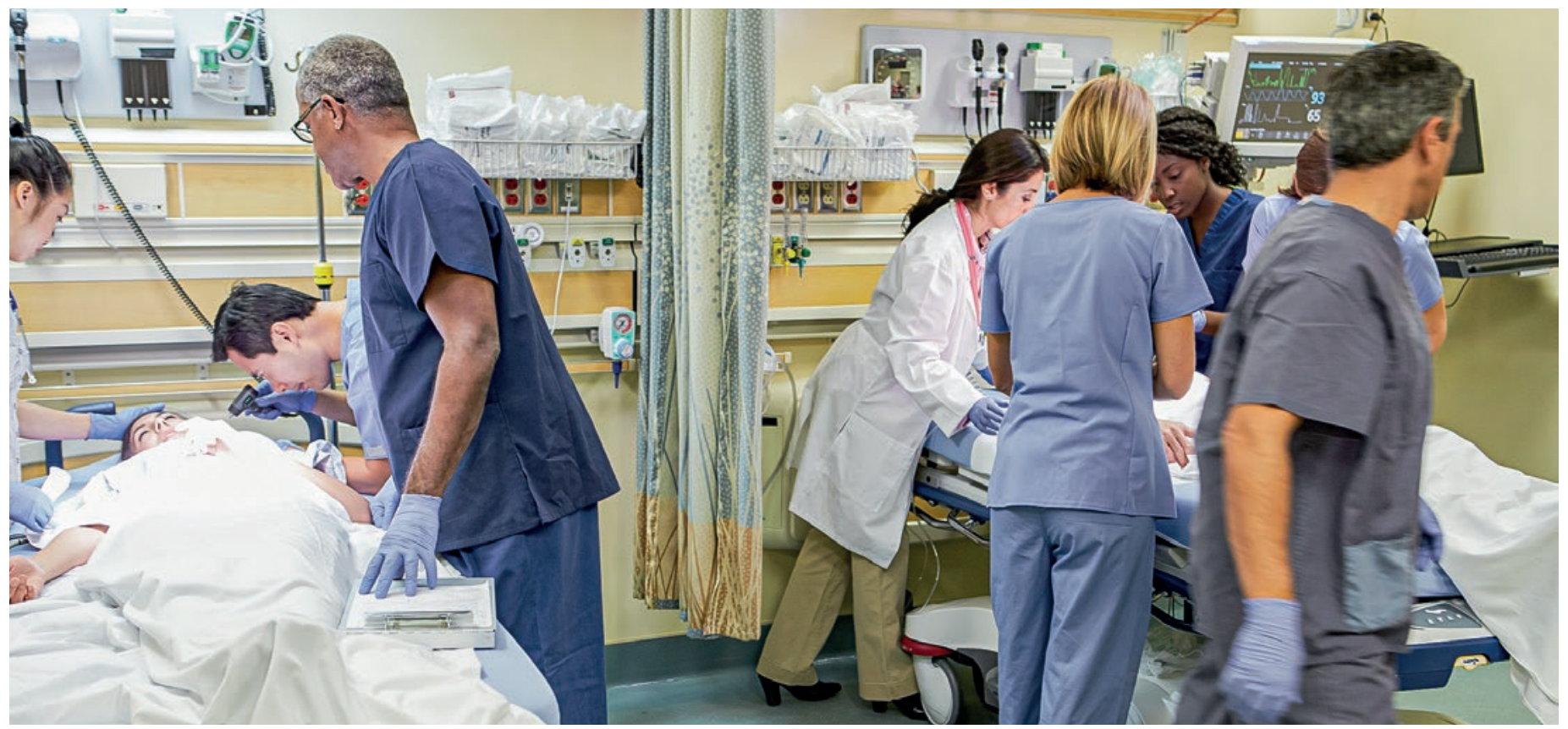

\title{
Erfolgreicher Beginn für eine gemeinsame Zukunft
}

\section{Cinzia Zeltner}

Wissenschaftliche Mitarbeiterin, Stv. Leiterin Sektion Weiterentwicklung Gesundheitsberufe, Bundesamt für Gesundheit (BAG)

Der Abschluss des Förderprogramms Interprofessionalität jährt sich dieser Tage zum ersten Mal. Was haben wir daraus gelernt? Wie hat das Bundesamt für Gesundheit (BAG) die Thematik weiterverfolgt? Und vor allem: Welche Schritte stehen als Nächstes an?

Ein zentrales Ziel des Förderprogramms Interprofessionalität war die Praxisrelevanz seiner Produkte. Die Resultate der Forschungsprojekte sollten nicht nur wissenschaftliche Grundlagen liefern, sondern auch Einzug in den Berufs- und Bildungsalltag finden. Dieses Ziel wurde im Grossen und Ganzen erreicht. Die Toolbox «Take Care» zum Thema psychische Gesundheit, die von der Zürcher Hochschule für angewandte Wissenschaften (ZHAW) erarbeitet wurde und Informationen für Fachpersonen, eine Broschüre für Jugendliche sowie einen Flyer für Kinder und deren Erziehungsberechtigte umfasst, erfreut sich zum Beispiel solch grosser Beliebtheit, dass die ursprüngliche Auflage bereits nachgedruckt werden musste.

Auch das vom Institut für Medizinische Lehre (IML) Bern und der Berner Fachhochschule (BFH) entwi- ckelte und validierte Schweizerische Interprofessionalitäts-Evaluationsinstrument (SIPEI) fand bereits Anwendung in der Praxis. Mit dem SIPEI können Institutionen erfassen, wie stark ihr Arbeitsumfeld auf Interprofessionalität ausgerichtet ist. Es beinhaltet Fragebögen für Mitarbeitende, Leitungspersonen sowie Patientinnen und Patienten.

\section{Modelle guter Praxis sichtbar machen}

Nebst den Forschungsprojekten wurde im Förderprogramm auch ein Verzeichnis mit Modellen guter Praxis finanziert. Die Idee dahinter war simpel: In der Schweiz wird Interprofessionalität bereits an vielen Orten gelebt. Oft sind diese Modelle aber nur regional bekannt. Mit dem Verzeichnis sollten sie gebündelt, schweizweit 
sichtbar gemacht und der Austausch zwischen interessierten Personen gefördert werden. Einige der Modelle wurden in zwei Broschüren im Detail porträtiert, um Interessierten einen vertieften Einblick in ihre Funktionsweise zu geben.

Ein Blick in das Verzeichnis lohnt sich, denn es deckt eine grosse Spannbreite an Themen, Settings und Berufsgruppen ab. Viele der erfassten Modelle haben zum Ziel, die Zusammenarbeit zwischen den Fachpersonen zu optimieren, damit sich diese stärker auf ihre Kerntätigkeiten konzentrieren und die Versorgungsqualität erhöhen können. Ein Beispiel ist das Projekt zum Einsatz von Advanced Practice Nurses in der Hausarztpraxis Bürglen, das vom Kanton Uri unterstützt und durch das Institut für Hausarztmedizin und Community Care Luzern wissenschaftlich evaluiert wurde. Auch das Modell des psychiatrischen Dienstes

\section{Modelle guter Praxis sind oft nur regional bekannt. Ein Verzeichnis soll sie bündeln, sichtbar machen und den Austausch fördern.}

Emmental zeigt, wie durch interprofessionelle Fallführung die Prozesse und die Versorgung optimiert werden können.

Andere Einträge sind in der Bildung und Lehre angesiedelt, beispielsweise die Module zur interprofessionellen Fallbesprechung des Universitätsspitals Zürich, welche Studierende der Medizin, Pflege, Geburtshilfe, Physiotherapie, Ergotherapie, Ernährungsberatung und Soziale Arbeit absolvieren. Es sind aber auch Einträge zu Instrumenten vorhanden, wie das Tool «SisCare», das Hausärztinnen und Hausärzte, Pflege-

\section{Gemeinsame Themenstränge}

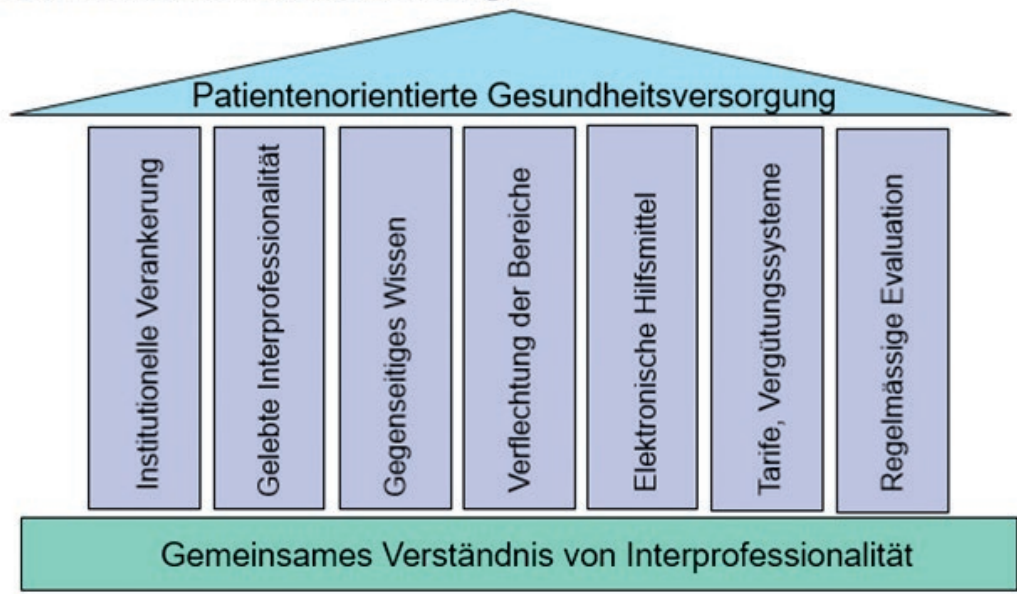

Künftige Handlungsfelder anhand von sieben Themensträngen. Quelle: BAG 2020. fachpersonen sowie Apothekerinnen und Apotheker nutzen können, um die Behandlung von chronisch kranken Menschen zu optimieren.

Das Verzeichnis ist ein langfristiges Engagement des BAG. Es wird laufend mit weiteren Einträgen ergänzt und Mitte 2022 in eine eigenständige Website migriert, welche gute Beispiele aus verschiedenen Bereichen des Gesundheitswesens umfassen und interessierten Personen Hilfestellungen zur Umsetzung eigener Projekte bieten wird.

\section{Weiterer Handlungsbedarf}

Obwohl das Förderprogramm bereits viele Forschungslücken schliessen konnte und der Praxistransfer insgesamt gelungen ist, so stellten die Arbeiten dennoch weiteren Handlungsbedarf fest. Dieser wurde in den vier Policy Briefs "Ambulante Versorgung", "Stationäre Versorgung», "Psychisch-somatische Nahtstelle» sowie "Bildung und Lehre» zusammengetragen. Er kann in sieben Themenstränge eingeteilt werden (vgl. Grafik). Künftige Massnahmen sollten demnach die institutionelle Verankerung von Interprofessionalität, das aktive Vorleben durch Führungspersonen, das gegenseitige Wissen über Rollen und Kompetenzen sowie die aktive Verflechtung der Akteure in den verschiedenen Bereichen des Gesundheitswesens fördern Sie sollten aber auch die Rahmenbedingungen wie die als ungenügend bewertete Funktionsweise elektronischer Hilfsmittel oder die tarifliche Abgeltung optimieren. Zudem wurde die regelmässige Evaluation der Umsetzung von Interprofessionalität, also die Anwendung von Tools wie dem SIPEI, als Handlungsfeld identifiziert.

\section{Einheitliches Verständnis schaffen}

Alle Themenfelder verfolgen als gemeinsames Ziel die bessere Versorgung von Patientinnen und Patienten. Damit dies erreicht werden kann, müssen künftige Massnahmen auf demselben Fundament aufbauen, konkret auf einem einheitlichen Verständnis von Interprofessionalität. Im Verlauf des Förderprogramms wurde klar, dass dies in der Schweiz noch nicht vorhanden ist. Aus diesem Grund liess das BAG Anfang 2021 durch das Büro Vatter eine Auslegeordnung erstellen, welche aufzeigte, wie die Interprofessionalität in der Literatur definiert ist und wie der Begriff in der Schweiz verwendet wird.

Dabei zeigte sich eine Diskrepanz zwischen Theorie und Praxis: Während die Interprofessionalität in der Theorie relativ klar von anderen Konzepten wie dem Task Shifting abgegrenzt ist, so wird der Begriff in der 
Praxis häufig synonym mit anderen verwendet. Dies ist im Hinblick auf das weitere Vorgehen relevant, denn für das BAG stellt sich die Frage, ob bei einer Förderung weiterer Projekte die wissenschaftliche Definition von Interprofessionalität zur Anwendung kommen soll oder ob der Begriff eher umgangssprachlich definiert bleiben müsste. Das BAG wird im Rahmen seines Strategieprozesses eine für die Schweiz gültige Definition von Interprofessionalität formulieren und Massnahmen daraus ableiten.

\section{Vernetzung fördern}

Ein weiterer Auftrag an das BAG, der aus den Policy Briefs hervorging, war die Bildung eines Netzwerks zur Stärkung der Koordination zwischen den Akteuren. Um zu klären, wie ein solches Netzwerk gestaltet werden könnte, führte das BAG im Frühjahr 2021 diverse Gespräche mit Stakeholdern. Dabei bestätigte sich, dass gerade im Bereich Bildung erst sehr lose Gefässe existieren und dass diese eher regional organisiert

Die Vernetzung zwischen Akteuren soll gestärkt werden. Das BAG ist bereit, im Bildungsbereich den Lead zu übernehmen.

sind. Aus den Gesprächen mit den Akteuren ging hervor, dass diese die Idee klar ablehnten, ein zusätzliches, übergeordnetes Netzwerk zu gründen. Vielmehr sahen sie es als zielführend, die bestehenden Strukturen besser miteinander zu vernetzen. Dabei äusserten sie mehrheitlich den Wunsch, der Bund solle diese Vernetzungsarbeit leisten. Das BAG ist bereit, den Lead in dieser Sache zu übernehmen und die Koordination zwischen bestehenden Netzwerken für interprofessionelle Bildung zu fördern. Gegen Mitte des Jahres wurden daher mögliche Massnahmen ausgearbeitet. Die konkrete Planung der weiteren Schritte verzögert sich jedoch, denn der Entscheid für viele dieser Massnahmen hängt davon ab, ob dem BAG in den nächsten Jahren weitere Gelder für die Unterstützung von Interprofessionalität in Form eines zweiten Förderprogramms zur Verfügung stehen werden oder nicht.

\section{Das Thema muss zentral bleiben}

Die Antwort auf diese Frage wird sich nicht zuletzt im Rahmen der Abstimmung zur Pflegeinitiative am 28. November 2021 ergeben. Wird die Initiative abgelehnt und tritt der indirekte Gegenvorschlag in Kraft, könnte das BAG ein zweites Förderprogramm lancieren. Denn mit dem indirekten Gegenvorschlag würden sowohl die gesetzlichen Grundlagen geschaffen, als auch finanzielle Mittel von bis zu fünf Millionen Franken zur Verfügung gestellt, um entsprechende Projekte zu unterstützen. Wird hingegen die Initiative angenommen, dann ist dies nicht der Fall. Projekte in der Grössenordnung eines Förderprogramms könnten in dem Szenario nicht umgesetzt werden, und auch die angedachten Massnahmen zur Förderung des Netzwerks Interprofessionalität müssten reduziert werden. Das BAG würde es sich zwar weiterhin zur Aufgabe machen, die Koordinationsarbeiten im Netzwerk zu übernehmen. Darüber hinaus gehende Investitionen wären jedoch nicht realistisch.

Unabhängig vom Ausgang der Abstimmung wird das Thema Interprofessionalität im Kontext der Strategie Gesundheit2030 zentral bleiben. Die erwähnten Koordinationsarbeiten im Netzwerk sowie die Integration der Modelle guter Praxis in eine externe Website sind zwei Beispiele dafür. Ausserdem könnte die Interprofessionalität bei einer allfälligen Revision der allgemeinen Lernziele und der Akkreditierung von ärztlichen Weiterbildungsgängen in den kommenden Jahren immer wieder aufgegriffen werden. Im Kontext der Plattform Zukunft ärztliche Bildung, die sich im nächsten Jahr intensiv mit der Frage des Arztbilds der Zukunft auseinandersetzen wird, will das BAG ebenfalls Wert darauf legen, die Interprofessionalität zu einem zentralen Thema zu machen. Zudem wird die interprofessionelle Versorgung im Rahmen der Massnahmen zur Kostendämpfung unterstützt, indem angedacht ist, Netzwerke zur koordinierten Versorgung als eigene Leistungserbringer zu definieren, was die Umsetzung von neuen Versorgungsmodellen erleichtern sollte. Ob darüber hinaus aber auch interprofessionelle Projekte und Vorhaben finanziert werden können, wird sich in wenigen Tagen zeigen.

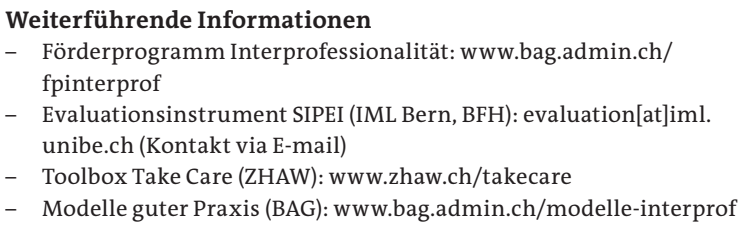

Bildnachweis

Monkey Business Images | Dreamstime.com

\section{Schwerpunktserie Interprofessionalität}

Die interprofessionelle Zusammenarbeit von Fachpersonen aus verschiedenen Gesundheitsberufen gilt als wichtiges Mittel, um den Herausforderungen im Gesundheitswesen zu begegnen. Aber wie weit ist die Schweiz in diesem Bereich tatsächlich? Welche Hürden und welche Chancen gibt es? In unserer Schwerpunktserie betrachten wir das Thema aus unterschiedlichen Perspektiven. 